\title{
APPLICATIONS OF GROUP ACTIONS ON FINITE COMPLEXES TO HILBERT CUBE MANIFOLDS
}

\author{
LEON STAGG NEWMAN, JR.
}

\begin{abstract}
For any compact Hilbert cube manifold $M$ such that $\tilde{H}_{*}\left(M, Z_{p}\right)$ $=0$, there exists an embedding $g$ of $M$ into the Hilbert cube $Q$ such that $g(M)$ is the fixed point set of a semifree periodic homeomorphism of $Q$ with period $p$. A counterexample is given to the conjecture that any two proper homotopic period $p$ homeomorphisms of a Hilbert cube manifold such that the homeomorphisms revolve trivially about a unique fixed point are equivalent. A counterexample is also given for the case where the fixed point set is empty.
\end{abstract}

I. Introduction. This paper continues the study of group actions on Hilbert cube $(Q)$ manifolds which essentially began with the work of R. Y. T. Wong [12]. That this study has begun in earnest is shown by the list of questions in [1]. Another recent paper in this area is [7].

The first part of this paper shows that certain finite-dimensional results generalize to $Q$ manifolds without the obstruction theory necessary in the finite-dimensional case.

The second part of this paper demonstrates that Wong's classification [12] of certain periodic homeomorphisms of $Q$ with unique fixed point does not generalize to $Q$ manifolds. Thus the theory of such actions on $Q$ manifolds should be richer than the theory of such actions on $Q$.

II. Definitions and notation. $Q=\Pi_{1}^{\infty}[-1,1]$, the Hilbert cube. A Hilbert cube manifold is a paracompact Hausdorff space admitting a cover of open sets homeomorphic to open sets of $Q$.

$f \simeq g$ means $f$ is homotopic to $g . f$ is properly homotopic to $g$ if there is a proper homotopy between $f$ and $g$. (A map is proper if the inverse image of every compact set is compact.)

$X \cong Y$ means $X$ is homeomorphic to $Y$. Simple homotopy theory will be used. See [6] for a general reference. $X \simeq_{s} Y$ means $X$ and $Y$ have the same simple homotopy type.

proj $\lim \left\{X_{i}, C_{i}\right\}=$ the inverse limit of the spaces $X_{i}$ with bonding maps $C_{i}$. In the following definitions, let $H$ be a homeomorphism of a space $X$ with

Presented to the Society, June 11, 1975 under the title Applications of group actions on finite complexes to $Q$ manifolds; received by the editors December 15,1975 .

AMS (MOS) subject classifications (1970). Primary 57E25, 57A20; Secondary 58B05, 54H15, $54 \mathrm{H} 25$.

Key words and phrases. Hilbert cube manifolds, semifree periodic homeomorphisms, fixed point set, simple homotopy equivalence, $\mathrm{CW}$-complex. 
period $p$; that is, $p$ is the smallest integer such that $H^{p}=\mathrm{id}$.

The fixed point set of $H$ is $\{x: h(x)=x\}$.

$H$ is semifree if for all $x$ such that

$$
H(x) \neq x, \quad x, \quad H(x), H^{2}(x), \ldots, H^{p-1}(x)
$$

are $p$ distinct points.

$H$ revolves trivially about its fixed point set $K$ if for every neighborhood $U$ of $K$, there is a contractible neighborhood $V \subset U$ of $K$ such that $H(V)=V$.

$X /\{H\}=$ the quotient of $X$ by the group generated by $H$, i.e., $X / \sim$ where $x \sim H^{i}(x), 1 \leqslant i \leqslant p-1$, for all $x \in X$.

$X /\left\{H\left(x_{0}\right)\right\}=X / \sim$ where $x_{0} \sim H^{i}\left(x_{0}\right), 1 \leqslant i \leqslant p-1$.

Two period $p$ homeomorphisms $f_{1}$ and $f_{2}$ of the space are equivalent if there exists a homeomorphism $g$ such that $f_{2}=g f_{1} g^{-1}$.

\section{III. $Q$ manifolds as fixed point sets.}

THEOREM 1. Let $M$ be a compact $Q$ manifold satisfying $\tilde{H}_{*}\left(M, Z_{p}\right)=0$ (the reduced singular homology with coefficients in the integers modulo $p$ ). There is an embedding $\mathrm{g}: M \rightarrow Q$ and a semifree, periodic homeomorphism $H: Q \rightarrow Q$ of period p such that $M$ is exactly the fixed point set of $H$.

Proof. Lowell Jones [9] proved that if $K$ is a finite CW-complex satisfying $H_{*}\left(K, Z_{p}\right)=0$, then there exists a finite contractible CW-complex $X$ containing $K$ as a subcomplex and a semifree, period $p$ homeomorphism $g: X \rightarrow X$ such that $K$ is the fixed point set of $g$.

Now T. A. Chapman's Triangulation Theorem for $Q$ manifolds [5] states that if $M$ is a compact $Q$ manifold, then there exist $K$, a finite simplicial (hence CW-) complex, and a homeomorphism $f: M \rightarrow K \times Q$. So $\tilde{H}_{*}\left(M, Z_{p}\right)$ $=\tilde{H}_{*}\left(K, Z_{p}\right)$.

For this $K$, let $h$ and $X$ be as in the conclusion to Jones' result. Let $i: K \rightarrow X$ be the inclusion map. Let $g=i \times$ id: $K \times Q \rightarrow X \times Q$. Let $H=$ $h \times$ id: $X \times Q \rightarrow X \times Q$. By a theorem of J. E. West [11], $X \times Q \cong Q$. So $g$ is the desired embedding and $H$ is the desired semifree periodic homeomorphism of period $p$.

IV. Counterexamples. R. Y. T. Wong [12] proved that if $f, g$ are semifree period $p$ homeomorphisms on $Q$ with a unique fixed point and revolve trivially about the fixed point, then $f$ and $g$ are equivalent. In [1], it was asked: If $f$ and $g$ are semifree period $p$ homeomorphisms of a $Q$ manifold $M$ such that (1) they have the same fixed point set $K$ and it is a single point or empty, (2) both $f$ and $g$ revolve trivially at $K$, and (3) $f$ is properly homotopic to $g$; then is $f$ equivalent to $g$ ?

The answer to both cases, $K=\varnothing$ and $K$ a single point, is no. The counterexample for the case $K=\varnothing$ is a straightforward construction using lens spaces [6, Chapter V]. The counterexample for the second case will take a bit more work.

Counterexample 1 . Let $S^{3}=\Sigma_{1} * \Sigma_{2}=$ the join of two copies of 


$$
S^{1}=\left\{\left(t e^{i x},(1-t) e^{i y}\right) \in R^{2} \times R^{2}: 0 \leqslant x, y \leqslant 2 \pi, 0<t<1\right\} .
$$

Let $f_{1}: S^{3} \rightarrow S^{3}$ be defined by

$$
f_{1}\left(t e^{i x},(1-t) e^{i y}\right)=\left(t e^{i x+2 \pi / 7},(1-t) e^{i y+2 \pi / 7}\right) .
$$

Let $f_{2}: S^{3} \rightarrow S^{3}$ be defined by

$$
f_{2}\left(t e^{i x},(1-t) e^{i y}\right)=\left(t e^{i x+4 \pi / 7},(1-t) e^{i y+2 \pi / 7}\right) .
$$

Let $M=S^{3} \times Q$, be a $Q$ manifold.

Clearly $g$ can be defined such that $g: S^{3} \times I \rightarrow S^{3}, g_{0}=f_{1}, g_{1}=f_{2}$. So $f_{1} \simeq f_{2}$. Define $F_{i}: M \rightarrow M$ by $F_{i}=f_{i} \times$ id $Q, i=1,2$. Each $F_{i}$ is a semifree period 7 homeomorphism of $M$. Define $G: F_{1} \simeq F_{2}$ by $G=g \times$ id $_{Q}$. So conditions (1) and (3) of the question are met and (2) is vacuously satisfied. (The spaces involved are compact so all homotopies are proper.)

Suppose there exists $H$ such that $F_{2}=H F_{1} H^{-1}$. Consider the following commutative diagram where $h$ is the naturally defined homeomorphism induced by $H$ and $q_{i}: M \rightarrow M /\left\{F_{i}\right\}$ is a naturally defined quotient map, $i=1,2$.

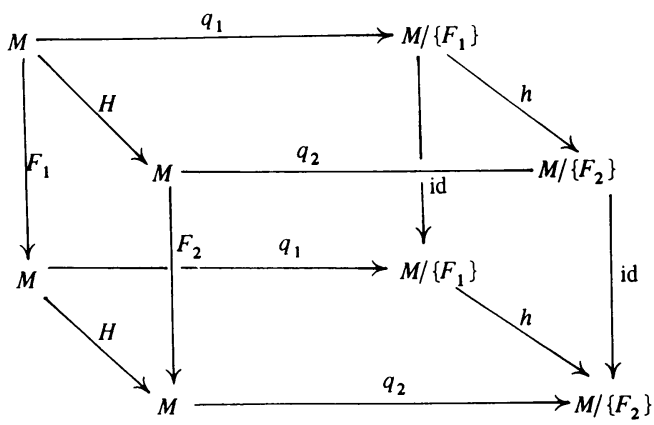

If $H$ existed, $M /\left\{F_{1}\right\} \cong M /\left\{F_{2}\right\}$. But $M /\left\{F_{i}\right\} \cong S^{3} /\left\{f_{i}\right\} \times Q, i=1,2$. By [6, §27], $S^{3} /\left\{f_{1}\right\} \cong L(7,1), S^{3} /\left\{f_{2}\right\} \cong L(7,2)$, where $L(p, q)$ is the standard 3-dimensional lens space.

Chapman's Classification Theorem [5] states that if $X$ and $y$ are compact, connected $Q$ manifolds and if $X \cong K \times Q$, and $Y \cong L \times Q(K, L$ finite simplicial complexes) are any two triangulations, then $X \cong Y$ if and only if $K$ and $L$ have the same simple homotopy type. But $L(7,1) \neq{ }_{s} L(7,2)[6, \S 31]$ and so $M /\left\{F_{1}\right\} \nRightarrow M /\left\{F_{2}\right\}$. So $H$ cannot exist and $f_{1}$ and $f_{2}$ are not equivalent.

Counterexample 2. Let $S^{3}, f_{1}, f_{2}$ be as in Counterexample 1. Let $T=$ $S^{3} /\left\{f_{1}^{i}\left(0, e^{i \pi(0)}\right)\right\}$. Note that $f_{1}^{i}\left(0, e^{i \pi(0)}\right)=f_{2}^{i}\left(0, e^{i \pi(0)}\right)$ for all $i$. Let $q: S^{3} \rightarrow T$ be the quotient map. From the definition of $T, f_{1}$, and $f_{2}, k_{1}=q f_{1} q^{-1}$ and $k_{2}=q f_{2} q^{-1}$ are well-defined semifree period 7 homeomorphisms of the CW-complex $T$.

Let $*$ denote the unique fixed point of the actions $k_{1}$ and $k_{2}$. Clearly $k_{1}$ and $k_{2}$ revolve trivially at $*$. Write $Q=\prod_{i=1}^{\infty} D_{i}$ where each $D_{i}$ is a two disk 
centered at the origin. Let $r_{i}: D_{i}^{2} \rightarrow D_{i}^{2}$ be the standard rotation of $D_{i}$ through $2 \pi / 7$ radians. Define $R: Q \rightarrow Q$ by $R\left(x_{1}, x_{2}, \ldots\right)=\left(r_{1}\left(x_{1}\right), r_{2}\left(x_{2}\right), \ldots\right)$ where $x_{i} \in D_{i}$ for all $i$. By a theorem of West [11], $T \times Q$ is a $Q$ manifold. Define $K_{i}=k_{i} \times R: T \times Q \rightarrow T \times Q, i=1$, 2. Each $K_{i}$ is a semifree period 7 homeomorphism revolving trivially around the same unique fixed point. $J=q g q^{-1} \times R$ is clearly a homotopy between $K_{1}$ and $K_{2}$ where $g$ is the homotopy from the previous example.

Now suppose $K_{1}$ was equivalent to $K_{2}$, that is there exists $P$ such that $K_{2}=P K_{1} P^{-1}$. Then $P$ would induce a homeomorphism $\bar{P}$ such that the following diagram would commute.

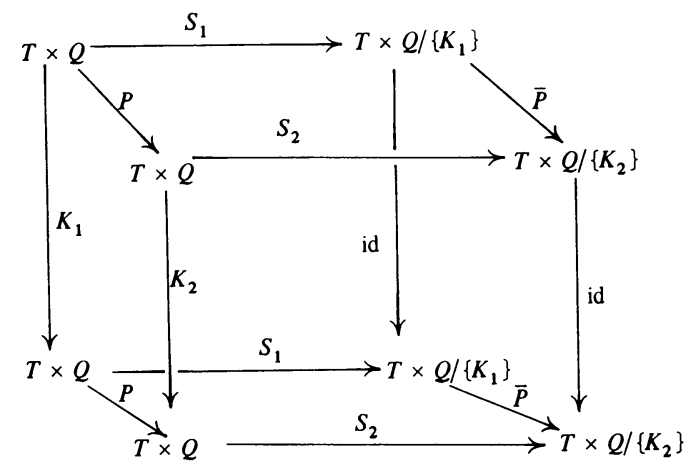

$S_{1}$ and $S_{2}$ are the naturally induced quotient maps. This would imply that $T \times Q /\left\{K_{1}\right\} \cong T \times Q /\left\{K_{2}\right\}$. But it will now be shown that $\left(T \times Q /\left\{K_{1}\right\}\right)$ $\times Q \cong L(7,1) \times Q$ and $\left(T \times Q /\left\{K_{2}\right\}\right) \times Q \cong L(7,2) \times Q$, and so $P$ cannot exist.

Define

$$
T_{0}=T /\left\{k_{1}\right\} \times(0,0, \ldots) \times Q
$$

and

$$
T_{n}=\frac{T \times \Pi_{i=1}^{n} D_{i}}{k_{1} \times \prod_{1}^{n} r_{i}} \times\left(0_{n+1}, 0_{n+2}, \ldots\right) \times Q
$$

for all $n \geqslant 1$.

Define $C_{n}: T_{n+1} \rightarrow T_{n}$ by

$$
C_{n}=\mathrm{id}_{T} \times \prod_{i=1}^{n} \operatorname{id}_{D_{i}} \times C_{n+1} \times \mathrm{id}
$$

where $C_{n+1}$ is induced by the radial collapse of $D_{n+1}$ to the origin. By the previously mentioned theorem of West, $T_{n}$ is a $Q$ manifold. Each $C_{n}$ is a $C E$-mapping and hence, by Chapman's $C E$-mapping Theorem [3], [4] (see [8] for a short proof), each $C_{i}$ is a near homeomorphism. So by Brown's Inverse Limit Theorem [2], proj $\lim \left\{T_{i}, C_{i}\right\} \cong T_{0}$. It is easy to put a metric on $T \times Q$ so that each $C_{i}$ is within $2^{-i}$ of the identity map. Since each $C_{i}$ is nonexpansive, the family $\left\{C_{n} \circ \cdots \circ C_{n+j}\right\} \cup_{j=0}^{\infty}$ is a uniformly equicontinuous family of maps. Certainly $\cup_{1}^{\infty} T_{i} \times Q$ is dense in $\left((T \times Q) /\left\{K_{1}\right\}\right) \times Q$. So by a lemma of R. Schori and D. Curtis [10], $\left((T \times Q) /\left\{K_{1}\right\}\right) \times Q \cong$ 
proj $\lim \left\{T_{i}, C_{i}\right\}$. So $\left((T \times Q) /\left\{K_{1}\right\}\right) \times Q \cong T_{0}$. But $T_{0}=\left(T /\left\{k_{1}\right\}\right) \times$ $(0,0, \ldots) \times Q \cong L(7,1) \times Q$. The proof that $\left((T \times Q) /\left\{K_{2}\right\}\right) \times Q \cong$ $L(7,2) \times Q$ is obviously identical. So $K_{1}$ and $K_{2}$ are not equivalent.

\section{REFERENCES}

1. R. D. Anderson and Nelly Kroonenberg, Open problems in infinite dimensional topology (preprint).

2. M. Brown, Some applications of an approximation theorem for inverse limits, Proc. Amer. Math. Soc. 11 (1960), 478-483. MR 22 \#5959.

3. T. A. Chapman, Cell-like mappings of Hilbert cube manifolds: Applications to simple homotopy theory (preprint).

4. , Cell-like mappings of Hilbert cube manifolds: Solution of a handle body problem (preprint).

5. Compact Hilbert cube manifolds and the invariance of Whitehead torsion, Bull. Amer. Math. Soc. 79 (1973), 52-56. MR 49 \#11518.

6. M. M. Cohen, $A$ course in simple-homotopy theory, Springer-Verlag, New York, 1973. MR 50 \#14762

7. D. A. Edwards, H. M. Hastings and J. West, Group actions on infinite dimensional manifolds (preprint).

8. A. Fathi, A cell-like map between Hilbert cube manifolds is a near homeomorphism, a short proof (preprint).

9. L. E. Jones, The converse to the fixed point theorem of P. A. Smith. I, Ann. of Math. (2) 94 (1971), no. 1, 52-68. MR 45 \#4427.

10. R. M. Schori and D. W. Curtis, Hyperspaces of compact connected polyhedra, Notices Amer. Math. Soc. 19 (1972), no. 141, A-809. Abstract \#699-G22.

11. J. E. West, Mapping cylinders of Hilbert cube factors, General Topology and Appl. 1 (1971), no. 2, 111-125. MR 44 \#5984.

12. R. Y. T. Wong, Periodic actions on the Hilbert cube (preprint).

Department of Mathematics, Baruch College, CUNY, New York, New York 10010

Current address: Bell Telephone Laboratories, Holmdel, New Jersey 07733 